\title{
The Compatibilization Effects of Alkylated-grafted-Graphene Oxide on Polypropylene/Polystyrene Blends
}

\author{
Yanping Hao, Xin Zhao, Jie Dong, and Qinghua Zhang \\ State Key Laboratory for Modification of Chemical Fibers and Polymer Materials, College of Material Science and Engineering, \\ Donghua University, Shanghai 201620, China
}

Correspondence should be addressed to Xin Zhao; xzhao@dhu.edu.cn and Qinghua Zhang; qhzhang@dhu.edu.cn

Received 7 December 2016; Accepted 5 February 2017; Published 8 March 2017

Academic Editor: Md M. Islam

Copyright (C) 2017 Yanping Hao et al. This is an open access article distributed under the Creative Commons Attribution License, which permits unrestricted use, distribution, and reproduction in any medium, provided the original work is properly cited.

\begin{abstract}
Modified graphene oxide (GO) was synthesized by covalently grafted alkylated chains on GO sheets and their compatibilization effects on the morphologies and mechanical properties of immiscible polypropylene/polystyrene (PP/PS) blends were investigated. Alkylated-grafted-GO/PP/PS batches were fabricated by melt-mixing approach and displayed different morphologies with various modified GO loadings. When the content of alkylated-grafted-GO is $0.2 \mathrm{wt} \%$, the tensile strength of obtained composite could reach 17.97 MPa, showing a 36.3\% enhancement compared to that of pristine PP/PS, indicating the positive compatibilization of modified GO in polymer blends. Moreover, the mixing order also plays an important role in achieving the desired improvement in properties. Due to the preferential location of modified GO in PP phase, a favorable "transition zone" could be formed at the interfacial region of two polymers when alkylated-grafted-GO was premixed with PS and subsequently mixed with PP, leading to an improvement of compatibilization between two polymers and an enhancement of mechanical properties. However, serious phase separation and declined tensile strength were obtained with a reversed mixing sequence.
\end{abstract}

\section{Introduction}

Blending of two or three polymeric materials is an effective way to produce new multiphase materials with excellent ultimate performances. The properties of the multiphase polymeric materials are usually affected by the properties of each component and the formed morphologies, especially near the interfacial regions. However, most of polymers are immiscible with each other because of the positive Gibbs energy of mixing, which results in serious phase separation, poor adhesion in interfaces, and deteriorated ultimate properties. One of the simplest and most effective ways to compatibilize immiscible polymers is to use appropriate compatibilizers such as graft or block copolymers $[1,2]$ and inorganic nanofillers such as clay or layered silicates [3-5], carbon nanotubes $[6,7]$, and graphene oxide $(\mathrm{GO})$ or its derivatives $[8,9]$.

The high modulus and low cost make inorganic nanofillers very attractive to compatibilize immiscible poly- mer blends. GO, as a product of the oxidative exfoliation of natural graphite, consists of multilayers of $\mathrm{sp}^{2}$-hybridized carbon atoms with a mixture of carboxyl, hydroxyl, and epoxy functional groups on the basal plane and the edges $[10,11]$. They can be easily decorated with strongly bound oxidative debris and have been applied in many applications, such as polymer composites, biosensors, and drug delivery systems $[12,13]$. Recently, GO and its derivatives are usually employed to compatibilize immiscible polymer blends and the $\pi-\pi$ stacking effects between $\mathrm{GO} /$ modified GO and aromatic rings of some polymers could be used to improve the interfacial interactions, resulting in an improved compatibility and better mechanical strength. Chemically coupling of polymethylmethacrylate (PMMA) on GO sheets (PMMA- $g$-GO) effectively improved the compatibility of immiscible polyvinylidenefluoride/acrylonitrile butadiene styrene (PVDF/ABS) blends, resulting in dramatic increments of $84 \%$ in Young's modulus and $124 \%$ in the yield strength for the blends compatibilized by PMMA-g-GO 
[14]. Modified GO sheets with polyethylene molecules (PE-g-GO) were adopted to reactively compatibilize blends of low density polyethylene and polyethylene oxide (LDPE/PEO), which led to a uniform dispersion of PEO in LDPE matrix and resulted in a significant improvement in the mechanical properties [15]. Polypropylene-graftedreduced graphene oxide (PP- $g$-rGO) was also reported as a novel compatibilizer for polypropylene/polystyrene (PP/PS) immiscible polymer blends and the strong interactions of PP$g$-rGO between PP and PS chains lead to the enhancements of the tensile strength and elongation at break of the PP/PS blends [16]. Thus, GO/modified GO in polymer blends could not only serve as compatibilizers but also act as reinforcing fillers due to their high modulus, which makes $\mathrm{GO}$ and its derivatives preferable to traditional copolymer compatibilizers.

Besides the surface modification of the filler and suitable compatibilizers, the processing technique also plays an important role in achieving the desired improvement in properties. The efficiency of the processing technique in dispersing the fillers becomes critical, especially with nanofillers, which have a very strong tendency to agglomerate due to their high surface energies or selective localization in the multiphase composites. Amani et al. [17] developed a new and effective strategy for compatibilizing immiscible polymer nanocomposites of polyethylene/PS/GO by a combination of solution intercalation and a melt-mixing method. Their studies showed that varying the mixing conditions resulted in different states of dispersion and different localizations of the fillers, leading to large discrepancies for the properties. Therefore, functionalization of appropriate compatibilizers and optimal processing technique are essential to influence the ultimate morphologies and properties of polymer blends $[18,19]$.

$\mathrm{PP} / \mathrm{PS}$ blends are typical immiscible polymer mixtures among plastics. Both of them have been widely used in many areas and played a significant role in polymer industry. But $\mathrm{PP} / \mathrm{PS}$ blends are immiscible due to the semicrystallinity of PP phase and make these polymer pairs chemically and mechanically incompatible. In our previous work, alkylated chains were successfully grafted to the surface of CNTs and the functionalized CNTs greatly improved the thermal stability and mechanical properties of polyimide matrix [20]. Meanwhile, alkylated-grafted-GO has also been confirmed to be good fillers to enhance the mechanical properties of polymers, like poly(lactic acid), polystyrene, and so forth [21, 22]. However, the compatibilization effects of alkylated-graftedGO on the polymer blends have not been reported (to our knowledge). In this study, alkylated chains $\left(-\mathrm{CONHC}_{18} \mathrm{H}_{37}\right)$ were covalently grafted on GO sheets through the reaction between carboxylic acid groups $(-\mathrm{COOH})$ on GO and $\mathrm{CH}_{3}\left(\mathrm{CH}_{2}\right)_{17} \mathrm{NCO}$. Then the obtained modified GO sheets were applied as compatibilizers for PP/PS blends. The compatibilization effects of alkylated-grafted-GO on the morphologies and properties of PP/PS blends with various modified GO loadings were investigated. Furthermore, the alkylated-grafted-GO was either premixed with PP or PS to study the effects of mixing orders on the morphologies and tensile properties of composites.

\section{Experimental Section}

2.1. Materials. Graphite oxide (GO) was made by modified Hummer's method [23]. Octadecyl isocyanate $\left(\mathrm{CH}_{3}\left(\mathrm{CH}_{2}\right)_{17} \mathrm{NCO}\right)$ with a purity $>99 \%$ was supplied by Sigma-Aldrich. Polypropylene (T30S, MFI $=3 \mathrm{~g} / 10 \mathrm{~min}$ ) was purchased from Maoming Petrochemical Chemical and polystyrene (PS144C) was obtained from BASF, China. $\mathrm{N}, \mathrm{N}$-Dimethylformamide (DMF), toluene, and acetone were purchased from Sinopharm Chemical Reagent Co., Ltd. All chemicals were used without further purification.

2.2. Preparation of Alkylated-grafted-Graphene Oxide. Dried $\mathrm{GO}$ powder $(300 \mathrm{mg})$ and $\mathrm{CH}_{3}\left(\mathrm{CH}_{2}\right)_{17} \mathrm{NCO}(3.5 \mathrm{~g})$ were put into a $100 \mathrm{~mL}$ three-necked flask with $30 \mathrm{~mL}$ DMF as a medium and stirred for $24 \mathrm{~h}$ at room temperature under $\mathrm{N}_{2}$ atmosphere. The mixture was poured into toluene; then the sediments were centrifuged and washed by acetone several times. Then the final products of alkylated-grafted-GO (noted as $\mathrm{aGO}$ ) were obtained.

2.3. Fabrication of Alkylated-grafted-GO Compatibilized PP/PS Blends. The alkylated-grafted-GO compatiblized PP/ PS $(70: 30, \mathrm{w} / \mathrm{w})$ blends were fabricated by melt-mixing method. A certain amount of modified GO with PP and PS was mixed by using Haake MiniLab microcompounder at $190^{\circ} \mathrm{C}$ with a screw speed of $60 \mathrm{rpm}$ for $10 \mathrm{~min}$, yielding $\mathrm{aGO} / \mathrm{PP} / \mathrm{PS}$ blends with different modified GO loadings. The various feeding ratios of aGO are $0.2 \mathrm{wt} \%, 0.5 \mathrm{wt} \%, 1.0 \mathrm{wt} \%$, and $5.0 \mathrm{wt} \%$, and the samples are designated as aGO/PP/PS$0.2, \mathrm{aGO} / \mathrm{PP} / \mathrm{PS}-0.5, \mathrm{aGO} / \mathrm{PP} / \mathrm{PS}-1.0$, and aGO/PP/PS-5.0, respectively. Furthermore, two other blending batches with different mixing orders were fabricated: aGO was firstly premixed with $\mathrm{PP}$ in the microcompounder at $190^{\circ} \mathrm{C}$ for $10 \mathrm{~min}$ and subsequently mixed with PS for more $10 \mathrm{~min}$ to get products (PP/aGO)/PS; and the other one was fabricated by just inversing the adding orders of $\mathrm{PP}$ and $\mathrm{PS}$ and the obtained blends were noted as (PS/aGO)/PP. In both (PP/aGO)/PS and (PS/aGO)/PP systems, the ratio of modified GO in PP/PS $(70: 30, \mathrm{w} / \mathrm{w})$ blends was controlled as $0.2 \mathrm{wt} \%$ and $0.5 \mathrm{wt} \%$, which are noted as $(\mathrm{PP} / \mathrm{aGO}) / \mathrm{PS}-0.2$, (PS/aGO)/PP-0.5, (PP/aGO)/PS-0.2, and (PS/aGO)/PP-0.5, respectively.

2.4. Measurements. The morphology and microstructure of the samples were investigated by field emission scanning electron microscope (FESEM, HitachiS-8000) and transmission electron microscope (TEM, JEM-2100). Fourier transform infrared (FTIR) spectra were recorded on a Nicolet 8700 FTIR spectrometer and the spectra in the range of 2000$500 \mathrm{~cm}^{-1}$ were collected by averaging 32 scans at a resolution of $4 \mathrm{~cm}^{-1}$. Raman spectra were conducted using a LabRam-1B Raman spectroscope with He-Ne laser excitation at $632.9 \mathrm{~nm}$ and scanning for $50 \mathrm{~s}$. For measurement of the mechanical properties of the composites, the sample were cut to sheets with a width of $10 \mathrm{~mm}$, and the measurement was conducted with XQ-1 instrument made in China. The lower grip was 


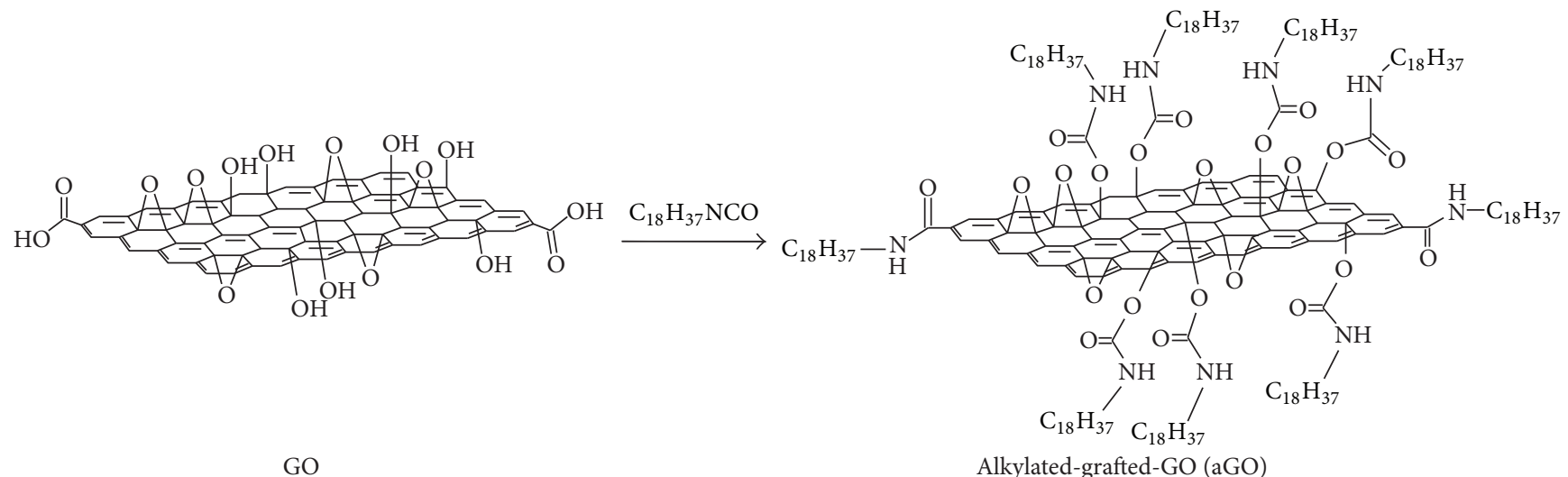

FIGURE 1: Schematic representation of GO sheets grafted with $\mathrm{CH}_{3}\left(\mathrm{CH}_{2}\right)_{17} \mathrm{NCO}$.

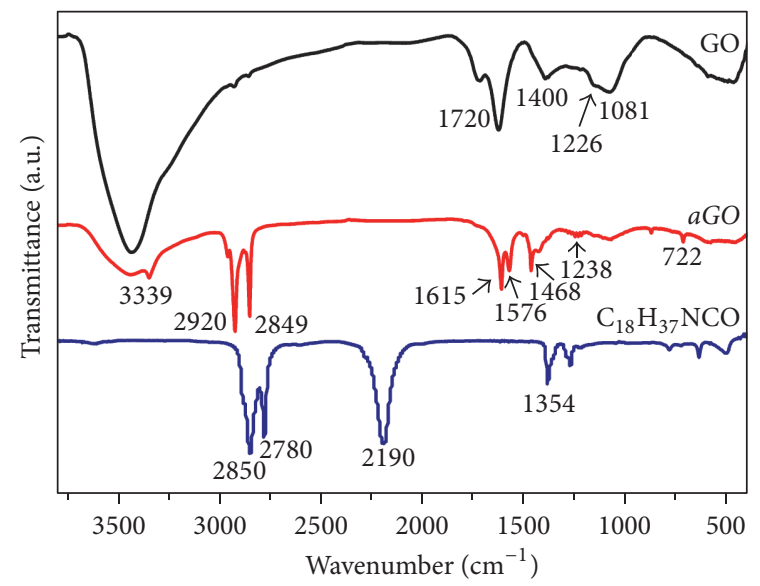

(a)

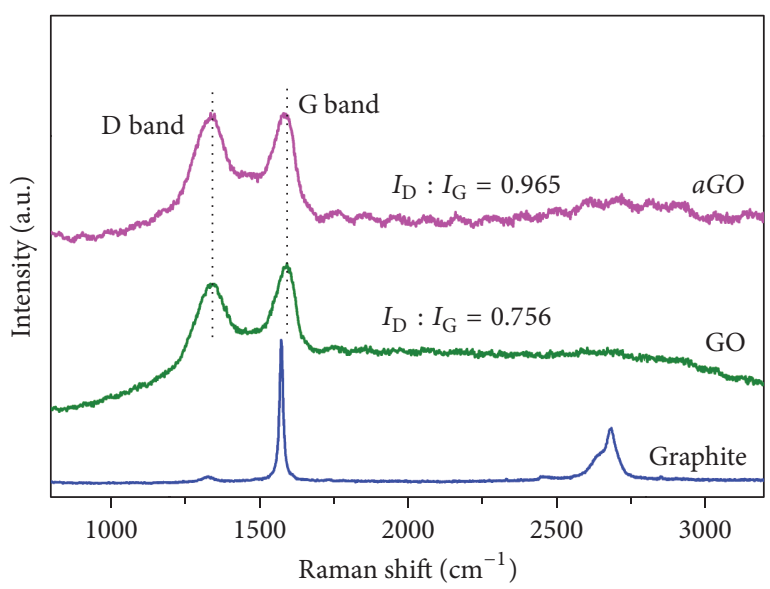

(b)

FIGURE 2: (a) FTIR spectra and (b) Raman spectra of GO, alkylated-grafted-GO (aGO), and $\mathrm{CH}_{3}\left(\mathrm{CH}_{2}\right)_{17} \mathrm{NCO}$.

fixed and the upper grip rose at a rate of $5 \mathrm{~mm} / \mathrm{min}$. The gauge length was $20 \mathrm{~mm}$.

\section{Results and Discussion}

3.1. Synthesis and Characterization of Alkylated-grafted-GO. It is known that graphene or graphene oxide nanosheets exhibit poor interactions with nonpolar PP chains but strong interactions with PS molecules through $\pi-\pi$ stacking [24]. Similar to our previous work [20], alkylated-grafted-GO was prepared through the reaction between carboxylic acid groups (-COOH) on $\mathrm{GO}$ and $\mathrm{CH}_{3}\left(\mathrm{CH}_{2}\right)_{17} \mathrm{NCO}$ as illustrated in Figure 1. The chemical structure and morphologies of obtained alkylated-g-GO were carefully characterized. Fourier transform infrared (FTIR) spectra were first carried out to characterize the chemical structures of modified GO and the spectra of $\mathrm{GO}, \mathrm{aGO}$, and $\mathrm{CH}_{3}\left(\mathrm{CH}_{2}\right)_{17} \mathrm{NCO}$ are shown in Figure 2(a). It has been reported that GO sheets consist of lots of oxygen groups such as hydroxyl and epoxy groups on the basal planes and carboxyl groups on the edges [13]. From the FTIR spectra, characteristic signals of GO are displayed at $\sim 1720 \mathrm{~cm}^{-1}, \sim 1400 \mathrm{~cm}^{-1}, \sim 1226 \mathrm{~cm}^{-1}$, and $\sim 1081 \mathrm{~cm}^{-1}$, which could be attributed to $\mathrm{C}=\mathrm{O}$ stretching mode in $-\mathrm{COOH}, \mathrm{O}-\mathrm{H}$ bending mode, $\mathrm{C}-\mathrm{OH}$ stretching mode, and $\mathrm{C}-\mathrm{O}$ stretching mode, respectively. The spectrum of aGO exhibits the new peaks at $3339 \mathrm{~cm}^{-1}$ and $1576 \mathrm{~cm}^{-1}$, corresponding to the $\mathrm{N}-$ $\mathrm{H}$ stretching and bending mode in amide group [20]. Other peaks of aGO at $\sim 1238 \mathrm{~cm}^{-1}, \sim 1468 \mathrm{~cm}^{-1}$, and $\sim 722 \mathrm{~cm}^{-1}$ could be ascribed to the $\mathrm{C}-\mathrm{N}$ stretching, $\mathrm{C}-\mathrm{H}$ stretching in alkylated chains, and $\mathrm{C}-\mathrm{C}$ stretching mode, respectively. Comparing with the line for $\mathrm{CH}_{3}\left(\mathrm{CH}_{2}\right)_{17} \mathrm{NCO}$, no signal of $\mathrm{N}=\mathrm{C}=\mathrm{O}$ peak at $\sim 2190 \mathrm{~cm}^{-1}$ appears for aGO, indicating the chemical reaction between - $\mathrm{COOH}$ groups on GO with -NCO . These observations clearly demonstrate that alkylated chains have been covalently bonded onto GO surfaces. Furthermore, Raman spectroscopy is also a powerful tool for characterizing the structure of graphene based materials. As shown in Figure 2(b), both aGO and GO show the $D$ band at $\sim 1350 \mathrm{~cm}^{-1}$ and $\mathrm{G}$ band at $\sim 1580 \mathrm{~cm}^{-1}$. The presence of $\mathrm{D}$ peak is associated with the defectiveness of the materials and the $G$ peak indicates the graphitized degree 


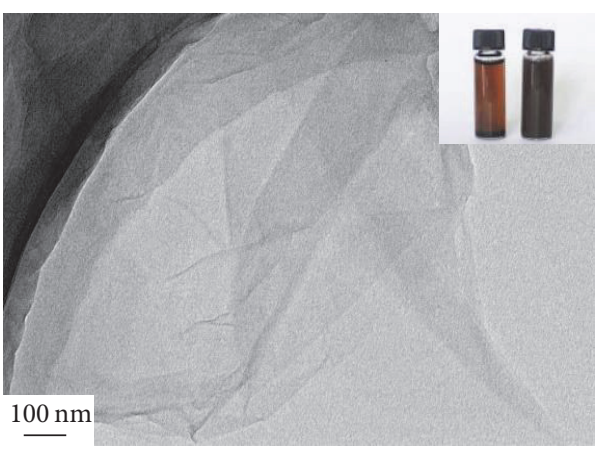

(a)

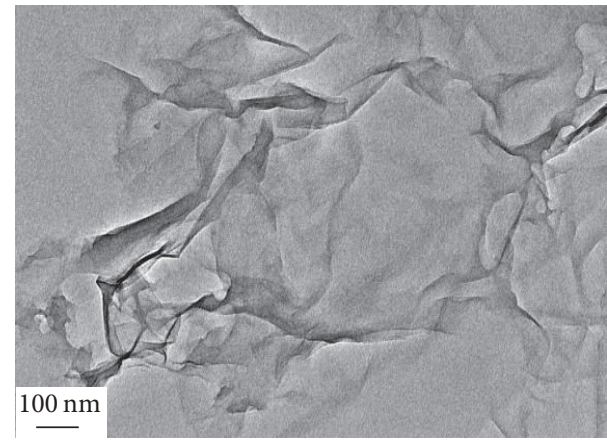

(b)

Figure 3: TEM images of (a) GO and (b) alkylated-grafted-GO (aGO).

TABLE 1: The elemental analysis results of GO and aGO.

\begin{tabular}{lccc}
\hline Sample & $\mathrm{C}(\mathrm{wt} \%)$ & $\mathrm{H}(\mathrm{wt} \%)$ & $\mathrm{N}(\mathrm{wt} \%)$ \\
\hline GO & 48.38 & 3.01 & $\leq 0.05$ \\
aGO & 73.34 & 11.62 & 3.78 \\
\hline
\end{tabular}

of carbon materials $[25,26]$. It is clearly seen that aGO shows a larger $I_{\mathrm{D}} / I_{\mathrm{G}}$ ratio than that of $\mathrm{GO}$, suggesting the increasing defects on aGO sheets after the reaction with $\mathrm{CH}_{3}\left(\mathrm{CH}_{2}\right)_{17} \mathrm{NCO}$.

TEM was also employed to observe the morphologies of dispersions of GO and aGO. As shown in Figure 3, the sheets of aGO could well remain the rippled nanosheets with wrinkles of GO sheets. The experimental observation shows that GO in dimethylformamide (DMF) easily agglomerates and settles to the bottom of a container within several days. But aGO can be uniformly dispersed in DMF after a couple of weeks, as shown in the inset of Figure 3(a). TEM image in Figure 3(b) also shows the modified graphene nanosheets with crumples due to its thin thickness. Moreover, the elemental analysis from Table 1 demonstrates an increase in content of $\mathrm{N}$ atoms in $\mathrm{aGO}$, further proving the existence of isocyanate groups on $\mathrm{GO}$ sheets.

3.2. Theoretical Prediction for the Localization of aGO in the $P P / P S$ Blends. The final localization of fillers in polymer blends is often determined by combination of thermodynamic and kinetic effects during the melt blending, and correct interpretations of these states need the knowledge about the thermodynamic driving force and practically processing parameters relating to aGO movements. The wetting coefficient $\left(\omega_{\alpha}\right)$ is often used to predict the thermodynamic location of fillers in polymer blends. Generally, the interfacial affinity between polymer components and inorganic fillers and the preferential localization of the fillers in the composites can be predicted by interfacial tensions between polymers and additives. According to Young's equation, $\omega_{\alpha}$ is proposed to define the location of aGO in an equilibrium state in PS/PP blends according to the following equation [27]:

$$
\omega_{\alpha}=\frac{\gamma_{\mathrm{aGO} / \mathrm{PP}}-\gamma_{\mathrm{aGO} / \mathrm{PS}}}{\gamma_{\mathrm{PP} / \mathrm{PS}}},
$$

where $\gamma_{\mathrm{aGO} / \mathrm{PP}}$ represents the interfacial tension between aGO and polymer component $\mathrm{PP}, \gamma_{\mathrm{aGO} / \mathrm{PS}}$ is the interfacial tension between aGO and PS, and $\gamma_{\mathrm{PP} / \mathrm{PS}}$ describes the interfacial tension between PP and PS. aGO is preferentially located in PS when $\omega_{\alpha}>1$, at the interface between the two phases when $-1<\omega_{a}<1$ and in PP component when $\omega_{a}<-1$.

The interfacial tension $\left(\gamma_{12}\right)$ can be determined from the surface energy and its disperse/polar components. It can be calculated according to the following two methods including Harmonic-mean and Geometric-mean equations. The former is valid between low-energy materials and the latter is often applied for a low-energy material and a high energy material $[28,29]$.

Harmonic-mean equation is as follows:

$$
\gamma_{12}=\gamma_{1}+\gamma_{2}-4\left(\frac{\gamma_{1}^{d} \gamma_{2}^{d}}{\gamma_{1}^{d}+\gamma_{2}^{d}}+\frac{\gamma_{1}^{p} \gamma_{2}^{p}}{\gamma_{1}^{p}+\gamma_{2}^{p}}\right)
$$

and Geometric-mean equation is as follows:

$$
\gamma_{12}=\gamma_{1}+\gamma_{2}-2\left(\sqrt{\gamma_{1}^{d} \gamma_{2}^{d}}+\sqrt{\gamma_{1}^{p} \gamma_{2}^{p}}\right)
$$

where $\gamma_{i}$ is the surface energy of component $i$ and $\gamma_{i}^{d}$ and $\gamma_{i}^{p}$ are the dispersive parts and polar parts of the surface tension of components, respectively.

The method used to determine the surface energy is based on the contact angle measurements between the liquid meniscus and the polymer surface. The geometric-mean method can be used to calculate the surface energy [30]:

$$
\gamma_{\mathrm{LV}}(1+\cos \theta)=2 \sqrt{\gamma_{\mathrm{SV}}^{d} \gamma_{\mathrm{LV}}^{d}}+2 \sqrt{\gamma_{\mathrm{SV}}^{p} \gamma_{\mathrm{LV}}^{p}},
$$

where $\theta$ is the contact angle, subscripts "LV" and "SV" denote the interfacial liquid-vapor and surface-vapor tensions, respectively, and superscripts " $d$ " and " $p$ " denote the 
TABLE 2: Surface energy data of PP, PS, and aGO.

\begin{tabular}{lccc}
\hline Samples & $\gamma\left(\mathrm{mN} \mathrm{m}^{-1}\right)$ & $\gamma^{d}\left(\mathrm{mN} \mathrm{m}^{-1}\right)$ & $\gamma^{p}\left(\mathrm{mN} \mathrm{m}^{-1}\right)$ \\
\hline PP & 15.93 & 13.40 & 2.53 \\
PS & 23.07 & 14.87 & 8.20 \\
aGO & 14.75 & 13.28 & 1.47 \\
\hline
\end{tabular}

TABLE 3: Interfacial tensions of component couples.

\begin{tabular}{lcc}
\hline Component couple & $\begin{array}{c}\text { Interfacial tension } \\
\left(\mathrm{mN} \mathrm{m}^{-1}\right) \\
\text { (harmonic-mean } \\
\text { equation) }\end{array}$ & $\begin{array}{c}\text { Interfacial tension } \\
\left(\mathrm{mN} \mathrm{m}^{-1}\right) \\
\text { (geometric-mean } \\
\text { equation) }\end{array}$ \\
\hline PP/PS & 3.07 & 1.66 \\
Modified graphene/PP & 0.28 & 0.14 \\
Modified graphene/PS & 4.77 & 2.77 \\
\hline
\end{tabular}

TABLE 4: The wetting coefficient values calculated by different equations.

\begin{tabular}{lcc}
\hline & $\begin{array}{c}\text { Harmonic-mean } \\
\text { equation }\end{array}$ & $\begin{array}{c}\text { Geometric-mean } \\
\text { equation }\end{array}$ \\
\hline Wetting coefficient $\left(\omega_{\alpha}\right)$ & -1.46 & -1.58 \\
\hline
\end{tabular}

disperse and polar components, respectively. Then the total surface tension $\left(\gamma_{\mathrm{SV}}\right)$ can be calculated by the equation

$$
\gamma_{\mathrm{SV}}=\gamma_{\mathrm{SV}}^{d}+\gamma_{\mathrm{SV}}^{p}
$$

Then the surface energy can be calculated by the contact angles of some representative liquids on the surface of polymer. Here, the selected representative liquids are distilled water $\left(\mathrm{H}_{2} \mathrm{O}\right)$ and ethylene glycol $\left(\left(\mathrm{CH}_{2} \mathrm{OH}\right)_{2}\right)$. The surface energy data of $\mathrm{H}_{2} \mathrm{O}$ and $\left(\mathrm{CH}_{2} \mathrm{OH}\right)_{2}$ are $\gamma^{d}=21.8 \mathrm{mN} \mathrm{m}^{-1}$ and $\gamma^{p}=51.0 \mathrm{mN} \mathrm{m}^{-1}$ for $\mathrm{H}_{2} \mathrm{O}$ and $\gamma^{d}=29.3 \mathrm{mN} \mathrm{m}^{-1}$ and $\gamma^{p}=19.0 \mathrm{mN} \mathrm{m}^{-1}$ for $\left(\mathrm{CH}_{2} \mathrm{OH}\right)_{2}$. The average contact angle between aGO and $\mathrm{H}_{2} \mathrm{O}$ is $107.12^{\circ}$ and that of aGO and $\left(\mathrm{CH}_{2} \mathrm{OH}\right)_{2}$ is $87.94^{\circ}$. Then, according to (4) and (5), the values of the surface energy of polymers are calculated and summarized in Table 2. All the results we used in this work are collected at room temperature.

As shown in Table 2, the surface energy of aGO is derived as $14.75 \mathrm{~m} \mathrm{~N} \mathrm{~m}^{-1}$, lower than the value of graphene in reported literatures [31,32], which might be attributed to the long alkylated chains grafted on graphene oxide surfaces [33, 34]. The interfacial tension between PP and aGO is much smaller than that of PS and aGO, indicating the discrepancy of aGO tendency in PP and PS blends. Then the interfacial tensions between each component can be calculated according to Harmonic-mean and Geometric-mean equations and the results are shown in Table 3. Finally, the wetting coefficient can be obtained and the values are -1.46 and -1.58 as displayed in Table 4, respectively. The low wetting coefficient $(<-1)$ indicates that aGO is preferentially located in the PP phase. However, kinetic migration of aGO plays a decisive role on the localization of aGO in the blends. The migration of aGO from one polymer bulk to another or to the interfacial areas is dependent on the diffusion, collisions, and Brownian motion of aGO in polymer bulk (PS or PP) as well as the viscoelastic properties of each polymer $[35,36]$.

3.3. Morphologies and Mechanical Properties of aGO/PP/PS Blends. To show the compatibilization effects of aGO on PP/PS blends, PP/PS (70/30, w/w) blends with different aGO contents were firstly fabricated through direct melt blending of all ingredients. The changes in phase morphologies of the samples as a function of aGO contents are depicted in Figure 4. For PP/PS (70:30) system, as shown in Figures $4(\mathrm{a})$ and $4\left(\mathrm{a}^{\prime}\right)$, obvious phase separation could be observed, indicating the incompatibility of two components. After adding aGO with a content of $0.5 \mathrm{wt} \%$ in Figures 4 (c) and $4\left(c^{\prime}\right)$, the PS domain size decreases and the large PS agglomerates gradually disappear when the loading of aGO reached $1.0 \mathrm{wt} \%$ as displayed in Figures $4(\mathrm{~d})$ and $4\left(\mathrm{~d}^{\prime}\right)$. Although the calculated results show a preferential location of aGO in PP, the FESEM observation clearly demonstrates the positive compatibilization effects of aGO nanosheets on $\mathrm{PP} / \mathrm{PS}$ blends. It is reported that the aGO sheets could absorb some PS chains on their graphene basal planes through $\pi-\pi$ interactions $[37,38]$. Meanwhile the theoretical calculation demonstrates that these functionalized nanosheets exhibit strong intermolecular interactions with PP phase, which might probably be due to the long alkylated chains grafted on their surfaces. Thus the aGO nanosheets tend to locate at the PP/PS interfacial regions to form a "transition zone" between polymer components, reducing unfavorable contacts and interracial tensions. Consequently, the compatibility between PP and PS is improved and the size of the dispersed phase domains decreases. However, when the content of aGO increases to $5 \mathrm{wt} \%$, aGO/PP/PS-5.0 shows a coarse crosssection surface as displayed in Figures $4(\mathrm{e})$ and $4\left(\mathrm{e}^{\prime}\right)$, which might be attributed to the increased PS domains and the aggregation of aGO sheets in the blends.

Generally, compatibilization can dramatically enhance the ultimate properties of immiscible polymer blends. The mechanical properties of PP/PS blends compatibilized by aGO with various loadings are given in Figure 5. The average tensile strength and tensile modulus of the PP/PS polymer blends are $13.19 \mathrm{MPa}$ and $2.70 \mathrm{GPa}$, respectively. The average tensile strength of aGO/PP/PS-0.2, aGO/PP/PS$0.5, \mathrm{aGO} / \mathrm{PP} / \mathrm{PS}-1.0$, and aGO/PP/PS-5.0 are $17.97 \mathrm{MPa}$, 16.52 MPa, 14.88 MPa, and 12.18 MPa, respectively, and their corresponding average tensile moduluses are $2.89 \mathrm{GPa}$, $2.68 \mathrm{GPa}, 2.65 \mathrm{GPa}$, and $2.64 \mathrm{GPa}$, respectively. It is obvious that the incorporation of aGO obviously improves the tensile strength and modulus of the composites, and the tensile strength of aGO/PP/PS- 0.2 reaches $17.97 \mathrm{MPa}$, showing a $36.3 \%$ enhancement compared to that of PP/PS. The possible reason for the improvement of mechanical properties might be the enhanced interfacial interactions among aGO and the two polymers. Besides, the reduced PS particle size caused by the addition of aGO also can promote the interfacial stress transfer. It should be mentioned that aGO can not only 


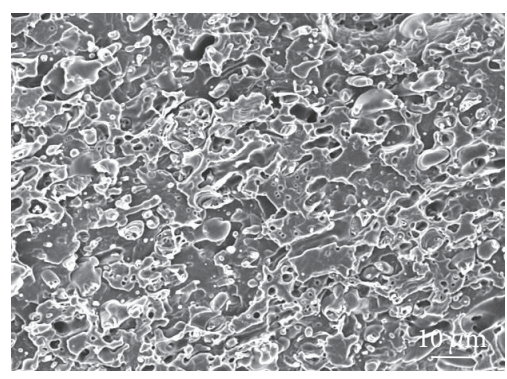

(a)

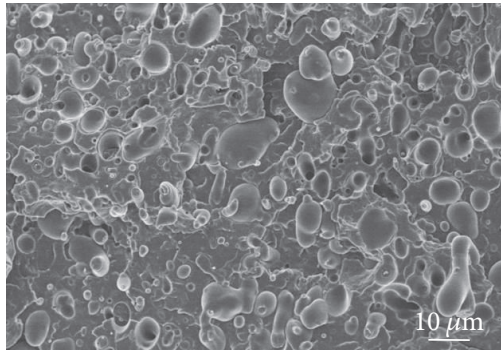

(b)

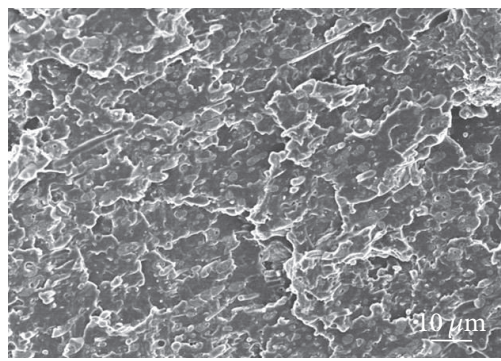

(c)

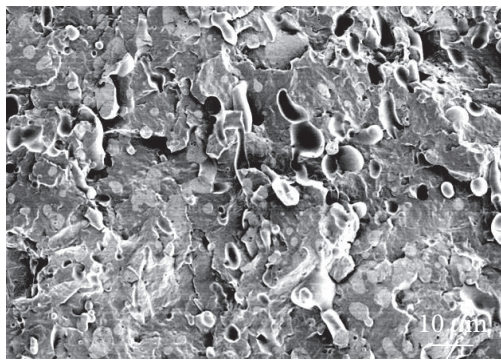

(d)

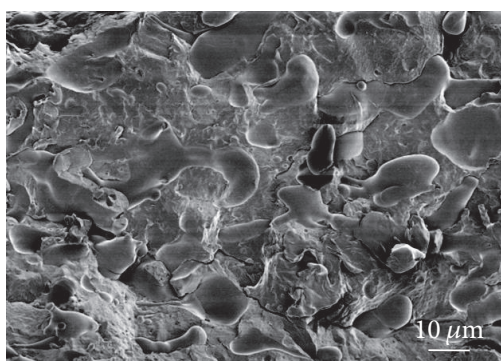

(e)

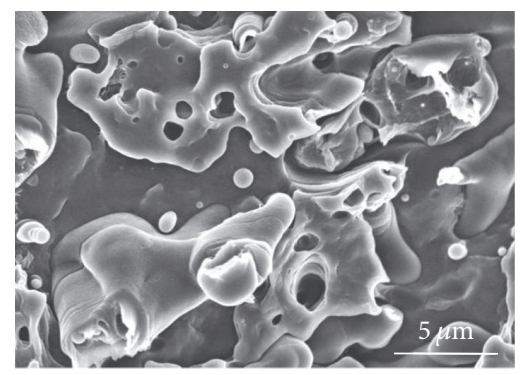

$\left(a^{\prime}\right)$

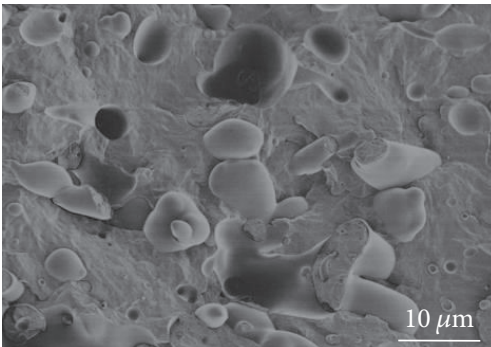

$\left(b^{\prime}\right)$

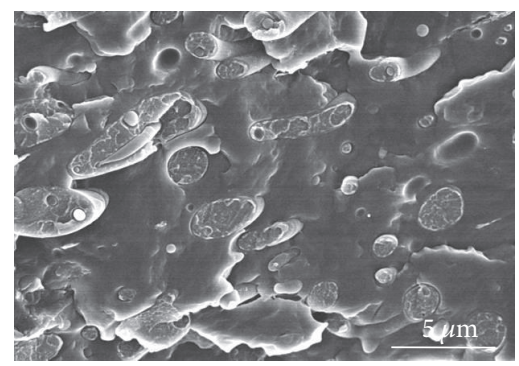

(c')

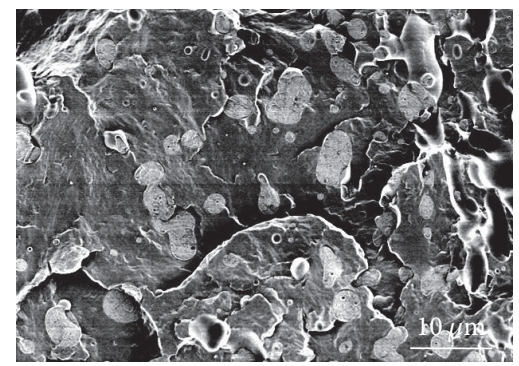

$\left(d^{\prime}\right)$

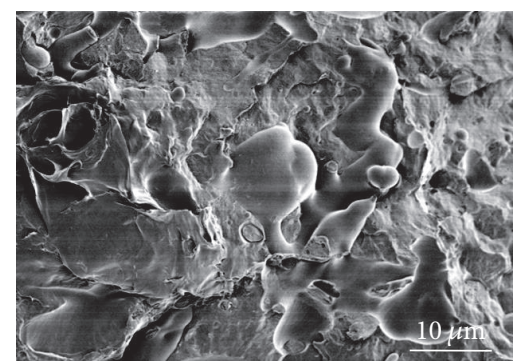

$\left(\mathrm{e}^{\prime}\right)$

FIGURE 4: The FESEM images of cross section of $\left[(\mathrm{a})\right.$ and $\left.\left(\mathrm{a}^{\prime}\right)\right] \mathrm{aGO} / \mathrm{PP} / \mathrm{PS}$, [(b) and $\left.\left(\mathrm{b}^{\prime}\right)\right] \mathrm{aGO} / \mathrm{PP} / \mathrm{PS}-0.5$, [(c) and $\left.\left(\mathrm{c}^{\prime}\right)\right] \mathrm{aGO} / \mathrm{PP} / \mathrm{PS}-1.0$, and $\left[(\mathrm{d})\right.$ and $\left.\left(\mathrm{d}^{\prime}\right)\right]$ aGO/PP/PS-5. 


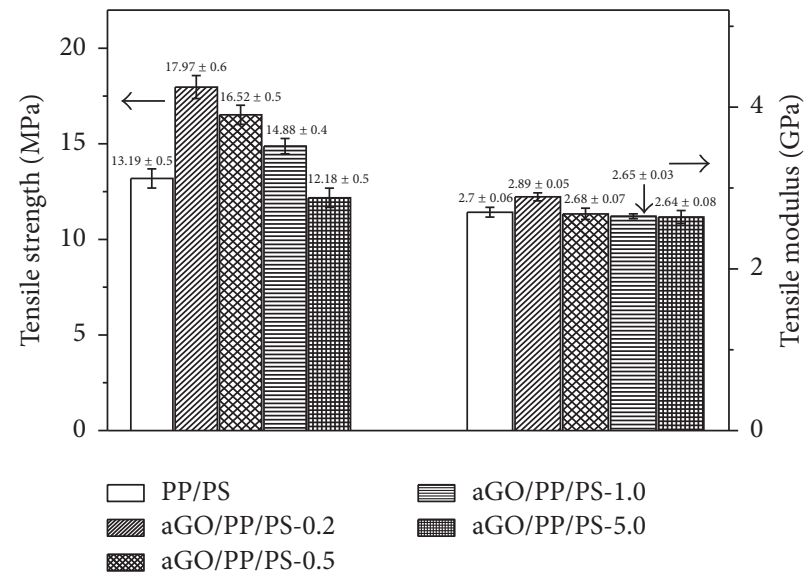

FIgURE 5: Tensile strength and tensile modulus of uncompatibilized PP/PS and aGO/PP/PS blends with various aGO loadings: aGO/PP/PS$0.2, \mathrm{aGO} / \mathrm{PP} / \mathrm{PS}-0.5$, aGO/PP/PS-1.0, and aGO/PP/PS-5.0.

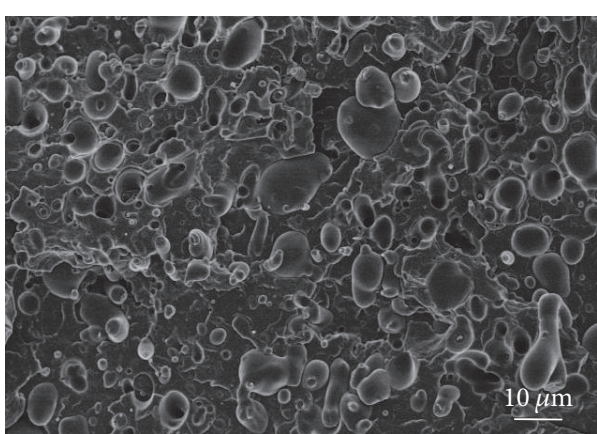

(a)

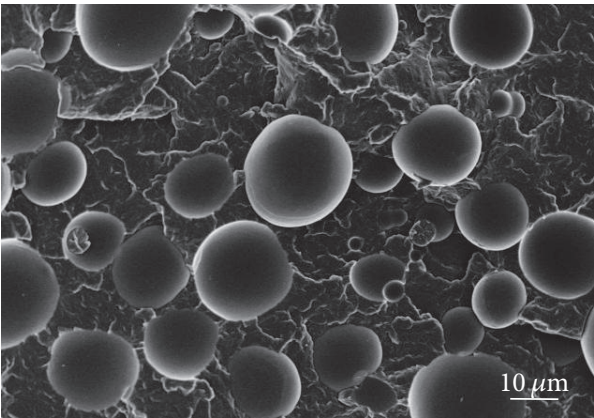

(b)

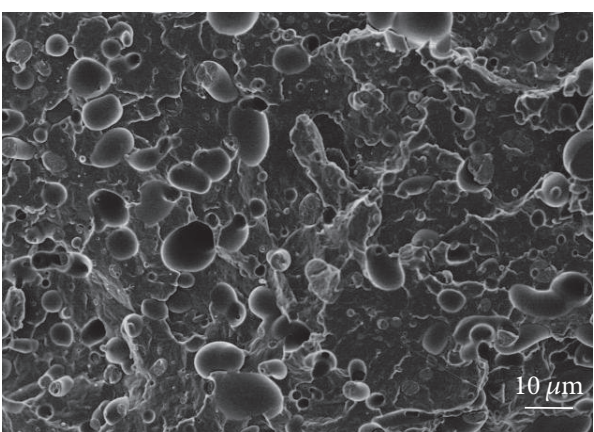

(c)

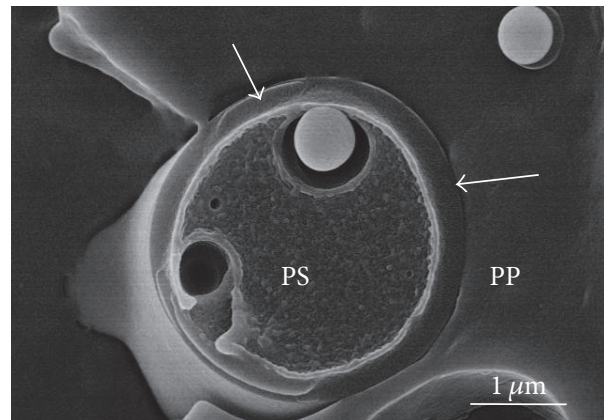

$\left(\mathrm{a}^{\prime}\right)$

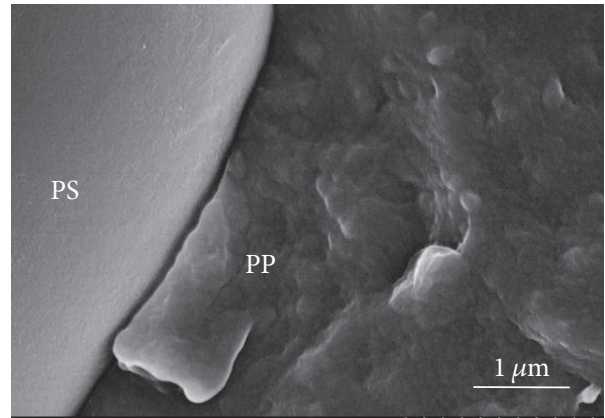

$\left(\mathrm{b}^{\prime}\right)$

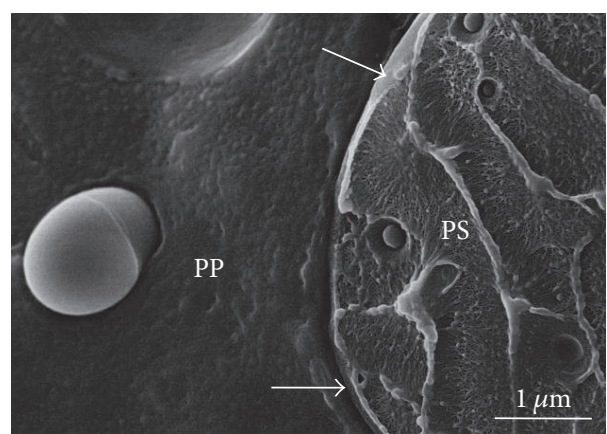

$\left(c^{\prime}\right)$

FIGURE 6: FESEM images showing the phase morphologies of $0.2 \mathrm{wt} \%$ aGO in representative composites. [(a) and (a')] aGO/PP/PS; [(b) and $\left.\left(\mathrm{b}^{\prime}\right)\right](\mathrm{PS} / \mathrm{aGO}) / \mathrm{PP} ;\left[(\mathrm{c})\right.$ and $\left.\left(\mathrm{c}^{\prime}\right)\right](\mathrm{PP} / \mathrm{aGO}) / \mathrm{PS}$ (the region pointed by arrows is the "transition zone"). 

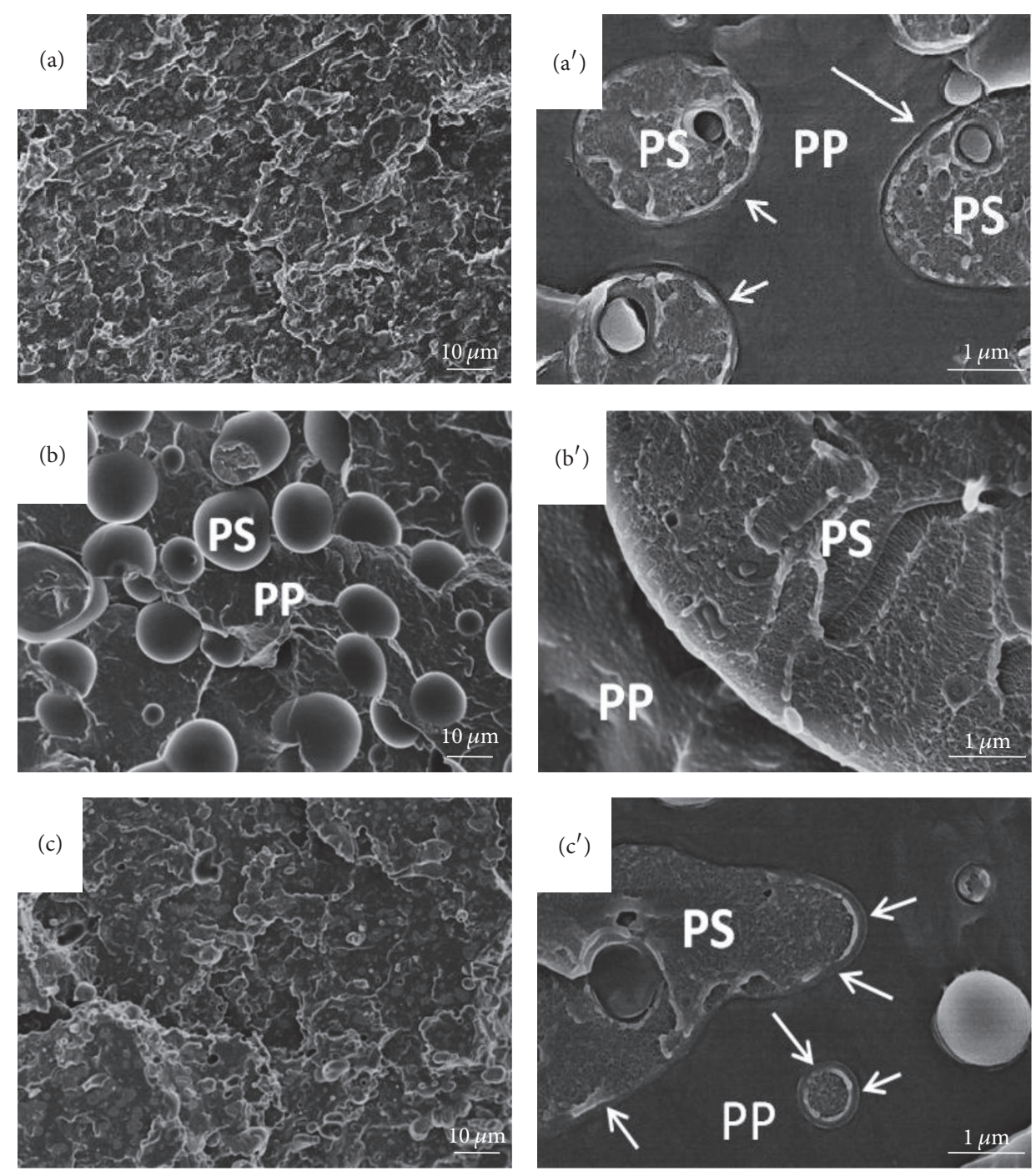

FIGURE 7: FESEM images showing the phase morphologies of $0.5 \mathrm{wt} \%$ aGO in representative composites. [(a) and (a')] aGO/PP/PS; [(b) and $\left.\left(\mathrm{b}^{\prime}\right)\right](\mathrm{PS} / \mathrm{aGO}) / \mathrm{PP} ;\left[(\mathrm{c})\right.$ and $\left.\left(\mathrm{c}^{\prime}\right)\right](\mathrm{PP} / \mathrm{aGO}) / \mathrm{PS}$.

have compatibilizing effect on PP/PS blends but also increase their stiffness because of the high modules of graphene, and then the tensile modulus of $\mathrm{aGO} / \mathrm{PP} / \mathrm{PS}$ increases to $2.89 \mathrm{GPa}$. However, when the feeding ratio of aGO rises higher than $0.5 \mathrm{wt} \%$, the tensile strength gradually declines and the modulus shows the similar level to that of pristine $\mathrm{PP} / \mathrm{PS}$ blends, which are consistent with the observation in the FESEM images in Figure 4.

3.4. Effects of Blending Orders on the Compatibilization of aGO in PP/PS Blends. To further explore the effects of blending orders on the localization of modified GO in PP/PS blends, two other different blends were prepared by controlling the mixing orders of aGO, PP, and PS (Experimental Section), which are denoted as (PP/aGO)/PS and (PS/aGO)/PP, respectively (the loading weight of aGO is $0.2 \mathrm{wt} \%$ and $0.5 \mathrm{wt} \%$ ). In Figure 6, compared with aGO/PP/PS-0.2 in (a) and $\left(\mathrm{a}^{\prime}\right),(\mathrm{PP} / \mathrm{aGO}) / \mathrm{PS}-0.2$ in $(\mathrm{b})$ and $\left(\mathrm{b}^{\prime}\right)$ displayed an obvious phase separation with a larger diameter of PS domains, which demonstrated obvious PS spheres, indicating the poor compatibilization of PP and PS. While (PS/aGO)/PP-0.2 in (c) and $\left(c^{\prime}\right)$ shows a more continuous morphology than those of (PP/aGO)/PS-0.2. Similar results also could be obtained for blending systems with $0.5 \mathrm{wt} \%$ loading of aGO as shown in Figure 7. Therefore, the mixing order has a great effect on the morphologies of final blends: when aGO is premixed with $\mathrm{PP}$ ((PP/aGO)/PS system), the preferential location of aGO in PP phase gives an unfavorable compatibilization effect on the PP/PS blends and induces the obvious phase separation; while when aGO is premixed with PS ((PS/aGO)/PP system), the selective location of aGO in PP drives it moving from PS phase into $\mathrm{PP}$, leading it to concentrate on the phase interface to form a "transition zone," which gives a positive influence on the compatibilization of PP and PS.

Furthermore, the tensile properties of PP/PS and aGO/PP/PS with different mixing orders are also investigated and given in Figure 8. The tensile strength and elongation 


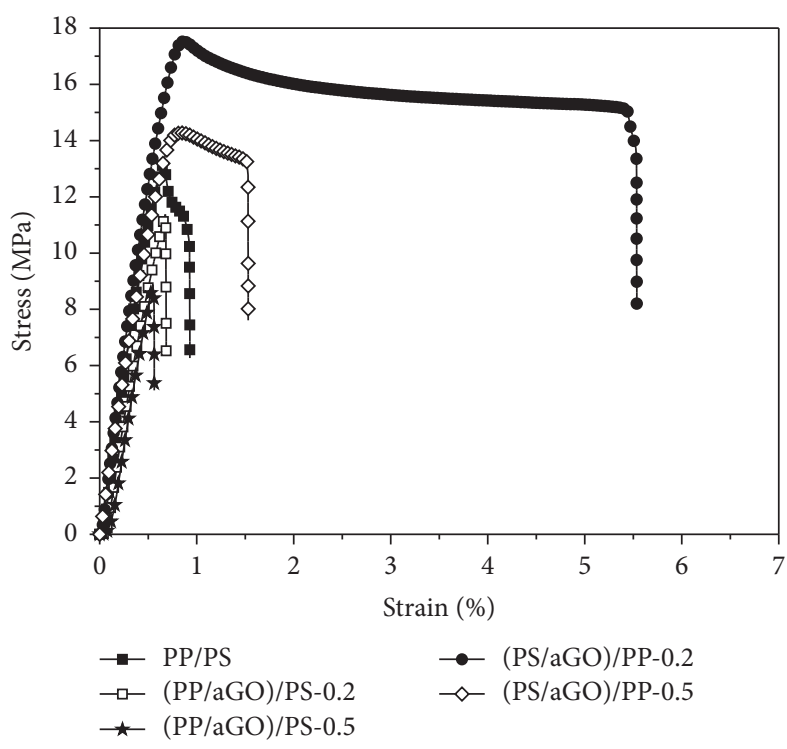

(a)

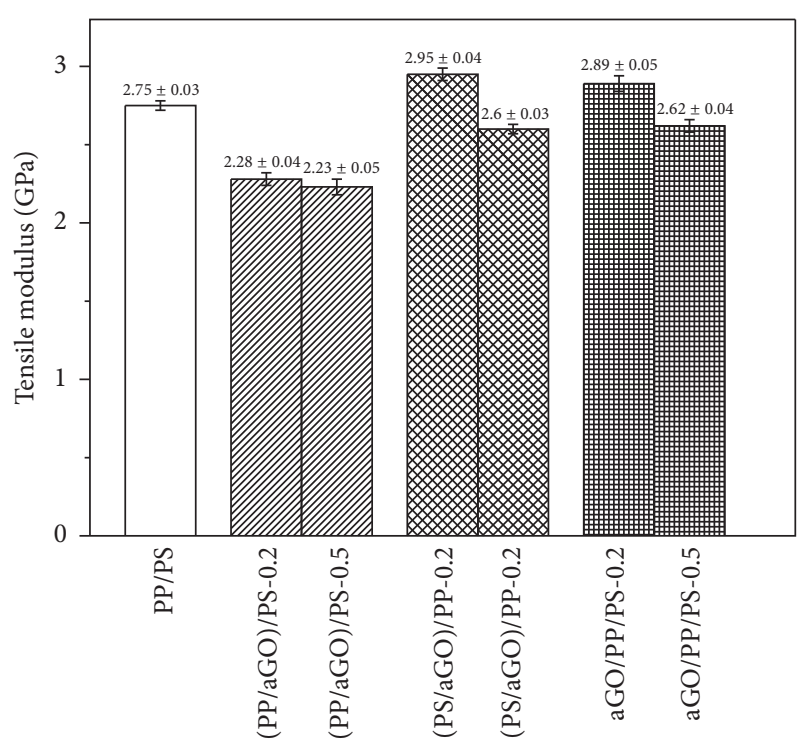

(b)

Figure 8: Stress-strain curves (a) and tensile modules (b) of (PS/aGO)/PP and (PP/aGO)/PS systems.

at break of the PP/PS polymer blends are $13.19 \mathrm{MPa}$ and $0.93 \%$, respectively. The tensile strength for $(\mathrm{PP} / \mathrm{aGP}) / \mathrm{PS}-0.2$, (PP/aGO)/PS-0.5, (PS/aGO)/PP-0.2, and (PS/aGO)/PP-0.5 is 11.42 $\mathrm{MPa}, 8.91 \mathrm{MPa}, 17.58 \mathrm{MPa}$, and $15.21 \mathrm{MPa}$, respectively. After adding alkylated-grafted-GO, (PP/aGP)/PS-0.2 and (PP/aGO)/PS- 0.5 show reduced mechanical properties with the tensile strength of $11.42 \mathrm{MPa}$ and $8.91 \mathrm{MPa}$, respectively, indicating the prior mixing of alkylated-grafted-GO with PP immobilizes the modified GO nanosheets in PP phase, resulting in the serious phase separation with declined mechanical properties. Meanwhile (PS/aGO)/PP-0.2 and (PS/aGO)/PP-0.5 displays an improved tensile strength of $17.58 \mathrm{MPa}$ and $15.32 \mathrm{MPa}$ with the elongation of $5.54 \%$ and $1.53 \%$, respectively, suggesting the enhanced interfacial interaction among modified GO loadings and the two polymers. Besides the compatibilizing effects, the modified GO also increases their stiffness because of high Young's modulus of graphene. As shown in Figure 8(b), with a loading of $0.2 \mathrm{wt} \%$ alkylated-grafted-GO, (PS/aGO)/PP displays the largest tensile modulus of $2.95 \mathrm{GPa}$, further confirming the selective localization of modified GO loadings with different mixing order and their great influence on the properties of PP/PS blends.

\section{Conclusions}

In conclusion, we successfully fabricated alkylated-graftedGO sheets by covalently grafting $\mathrm{CH}_{3}\left(\mathrm{CH}_{2}\right)_{17} \mathrm{NCO}$ on GO sheets and studied their compatibilization effects on the morphologies and mechanical properties of PP/PS blends. The results showed that an appropriate content of modified GO in PP/PS blends could be used as a favorable compatibilizer to improve the mechanical properties of PP/PS blends. With $0.2 \mathrm{wt} \%$ content of alkylated-grafted-GO, the tensile strength of aGO/PP/PS-0.2 could reach $17.97 \mathrm{MPa}$, showing a $36.3 \%$ enhancement compared to that of PP/PS. Besides, the mixing orders also greatly affect the morphologies and properties of polymer blends. When modified GO was premixed with PS and then mixed with PP, the obtained (PP/aGO)/PS batches displayed a more continuous morphology and a better mechanical property compared to those of (PS/aGO)/PP.

\section{Competing Interests}

The authors declare that they have no competing interests.

\section{References}

[1] A. A. Adewole, A. Denicola, C. G. Gogos, and L. Mascia, "Compatibilization of polypropylene-polystyrene blends: Part 2, crystallization behavior and mechanical properties," Advances in Polymer Technology, vol. 19, no. 3, pp. 180-193, 2000.

[2] C.-J. Hung, H.-Y. Chuang, and F.-C. Chang, "Novel reactive compatibilization strategy on immiscible polypropylene and polystyrene blend," Journal of Applied Polymer Science, vol. 107, no. 2, pp. 831-839, 2008.

[3] A. K. Singh, R. Prakash, and D. Pandey, "A comparative thermal, optical, morphological and mechanical properties studies of pristine and C15A nanoclay-modified PC/PMMA blends: a critical evaluation of the role of nanoclay particles as compatibilizers," RSC Advances, vol. 3, no. 35, pp. 15411-15420, 2013.

[4] B. Yalcin and M. Cakmak, "The role of plasticizer on the exfoliation and dispersion and fracture behavior of clay particles in PVC matrix: a comprehensive morphological study," Polymer, vol. 45, no. 19, pp. 6623-6638, 2004.

[5] C.-H. O. Chen, C.-C. Teng, and C.-H. Yang, "Preparation and characterization of rigid poly (vinyl chloride)/MMT nanocomposites," Journal of Polymer Science, Part B: Polymer Physics, vol. 43, no. 12, pp. 1465-1474, 2005.

[6] G. R. Kasaliwal, S. Pegel, A. Göldel, P. Pötschke Petra, and G. Heinrich, "Analysis of agglomerate dispersion mechanisms of 
multiwalled carbon nanotubes during melt mixing in polycarbonate," Polymer, vol. 51, no. 12, pp. 2708-2720, 2010.

[7] G. Kasaliwal, A. Göldel, and P. Pötschke, "Influence of processing conditions in small-scale melt mixing and compression molding on the resistivity and morphology of polycarbonateMWNT composites," Journal of Applied Polymer Science, vol. 112, no. 6, pp. 3494-3509, 2009.

[8] A. Pandikumar, G. T. Soon How, T. P. See et al., "Graphene and its nanocomposite material based electrochemical sensor platform for dopamine," RSC Advances, vol. 4, no. 108, pp. 63296-63323, 2014.

[9] R. K. Upadhyay, N. Soin, and S. S. Roy, "Role of graphene/metal oxide composites as photocatalysts, adsorbents and disinfectants in water treatment: a review," RSC Advances, vol. 4, no. 8, pp. 3823-3851, 2014.

[10] J. Kim, L. J. Cote, F. Kim, W. Yuan, K. R. Shull, and J. Huang, "Graphene oxide sheets at interfaces," Journal of the American Chemical Society, vol. 132, no. 23, pp. 8180-8186, 2010.

[11] Y. Cao, J. Zhang, J. Feng, and P. Wu, "Compatibilization of immiscible polymer blends using graphene oxide sheets," ACS Nano, vol. 5, no. 7, pp. 5920-5927, 2011.

[12] J. P. Rourke, P. A. Pandey, J. J. Moore et al., "The real graphene oxide revealed: stripping the oxidative debris from the graphene-like sheets," Angewandte Chemie-International Edition, vol. 50, no. 14, pp. 3173-3177, 2011.

[13] D. R. Dreyer, S. Park, C. W. Bielawski, and R. S. Ruoff, "The chemistry of graphene oxide," Chemical Society Reviews, vol. 39, no. 1, pp. 228-240, 2010.

[14] G. P. Kar, S. Biswas, and S. Bose, “Tailoring the interface of an immiscible polymer blend by a mutually miscible homopolymer grafted onto graphene oxide: outstanding mechanical properties," Physical Chemistry Chemical Physics, vol. 17, no. 3, pp. 1811-1821, 2015.

[15] P. K. S. Mural, A. Banerjee, M. S. Rana et al., "Polyolefin based antibacterial membranes derived from PE/PEO blends compatibilized with amine terminated graphene oxide and maleated PE," Journal of Materials Chemistry A, vol. 2, no. 41, pp. 17635-17648, 2014.

[16] F. You, D. Wang, X. Li, M. Liu, Z.-M. Dang, and G.-H. Hu, "Synthesis of polypropylene-grafted graphene and its compatibilization effect on polypropylene/polystyrene blends," Journal of Applied Polymer Science, vol. 131, no. 13, 2014.

[17] M. Amani, M. Sharif, A. Kashkooli, N. Rahnama, and A. Fazli, "Effect of mixing conditions on the selective localization of graphite oxide and the properties of polyethylene/highimpact polystyrene/graphite oxide nanocomposite blends," RSC Advances, vol. 5, no. 95, pp. 77723-77733, 2015.

[18] A. K. Krishnan and K. E. George, "Polymer blend nanocomposites: effect of mercapto silane modified kaolin clay on the thermal properties of Polypropylene/Polystyrene blend," Polymers for Advanced Technologies, vol. 25, no. 9, pp. 955-962, 2014.

[19] M. Liebscher, M.-O. Blais, P. Pötschke, and G. Heinrich, "A morphological study on the dispersion and selective localization behavior of graphene nanoplatelets in immiscible polymer blends of PC and SAN," Polymer, vol. 54, no. 21, pp. 5875-5882, 2013.

[20] Q. Zhang, J. Li, X. Zhao, and D. Chen, "Preparation and characterization of alkylated carbon nanotube/polyimide nanocomposites," Polymer International, vol. 58, no. 5, pp. 557-563, 2009.

[21] L. Zhang, Y. Li, H. Wang, Y. Qiao, J. Chen, and S. Cao, "Strong and ductile poly(lactic acid) nanocomposite films reinforced with alkylated graphene nanosheets," Chemical Engineering Journal, vol. 264, pp. 538-546, 2015.

[22] J. Jang, V. H. Pham, B. Rajagopalan, S. H. Hur, and J. S. Chung, "Effects of the alkylamine functionalization of graphene oxide on the properties of polystyrene nanocomposites," Nanoscale Research Letters, vol. 9, article no. 265, 2014.

[23] W. S. Hummers Jr. and R. E. Offeman, "Preparation of graphitic oxide," Journal of the American Chemical Society, vol. 80, no. 6, article 1339, 1958.

[24] C. Wan and B. Chen, "Reinforcement and interphase of polymer/graphene oxide nanocomposites," Journal of Materials Chemistry, vol. 22, no. 8, pp. 3637-3646, 2012.

[25] C. Gao, Y. Z. Jin, H. Kong et al., "Polyurea-functionalized multiwalled carbon nanotubes: synthesis, morphology, and Raman spectroscopy," Journal of Physical Chemistry B, vol. 109, no. 24, pp. 11925-11932, 2005.

[26] H. L. Zeng, C. Gao, and D. Y. Yan, "Poly ( $\varepsilon$-caprolactone)-functionalized carbon nanotubes and their biodegradation properties," Advanced Functional Materials, vol. 16, pp. 812-818, 2006.

[27] M. Sumita, K. Sakata, S. Asai, K. Miyasaka, and H. Nakagawa, "Dispersion of fillers and the electrical conductivity of polymer blends filled with carbon black," Polymer Bulletin, vol. 25, no. 2, pp. 265-271, 1991.

[28] S. Wu, Polymer Interface and Adhesion, M. Dekker, 1982.

[29] G. Yu, M. Q. Zhang, H. M. Zeng, Y. H. Hou, and H. B. Zhang, "Effect of filler treatment on temperature dependence of resistivity of carbon-black-filled polymer blends," Journal of Applied Polymer Science, vol. 73, no. 4, pp. 489-494, 1999.

[30] D. K. Owens and R. C. Wendt, "Estimation of the surface free energy of polymers," Journal of Applied Polymer Science, vol. 13, no. 8, pp. 1741-1747, 1969.

[31] S. Wang, Y. Zhang, N. Abidi, and L. Cabrales, "Wettability and surface free energy of graphene films," Langmuir, vol. 25, no. 18, pp. 11078-11081, 2009.

[32] M. Pérez-Mendoza, M. Almazán-Almazán, L. Méndez-Liñán, M. Domingo-García, and F. López-Garzón, "Evaluation of the dispersive component of the surface energy of active carbons as determined by inverse gas chromatography at zero surface coverage," Journal of Chromatography A, vol. 1214, no. 1-2, pp. 121-127, 2008.

[33] Y. Shen, T.-T. Zhang, J.-H. Yang, N. Zhang, T. Huang, and Y. Wang, "Selective localization of reduced graphene oxides at the interface of PLA/EVA blend and its resultant electrical resistivity," Polymer Composites, 2015.

[34] J. Chen, Y.-B. Hou, M.-L. Zhang et al., "Combined effect of compatibilizer and carbon nanotubes on the morphology and electrical conductivity of PP/PS blend," Polymers for Advanced Technologies, vol. 25, no. 6, pp. 624-630, 2014.

[35] R. Salehiyan, Y. Yoo, W. J. Choi, and K. Hyun, "Characterization of morphologies of compatibilized Polypropylene/ Polystyrene blends with nanoparticles via nonlinear rheological properties from FT-rheology," Macromolecules, vol. 47, no. 12, pp. 40664076, 2014.

[36] J. Parameswaranpillai, G. Joseph, R. V. Chellappan, A. K. Zahakariah, and N. Hameed, "The effect of polypropylenegraft-maleic anhydride on the morphology and dynamic mechanical properties of polypropylene/polystyrene blends," Journal of Polymer Research, vol. 22, no. 2, 2015.

[37] M. Yang, V. Koutsos, and M. Zaiser, "Interactions between polymers and carbon nanotubes: a molecular dynamics study," 
Journal of Physical Chemistry B, vol. 109, no. 20, pp. 1000910014, 2005.

[38] M. Fang, K. Wang, H. Lu, Y. Yang, and S. Nutt, "Singlelayer graphene nanosheets with controlled grafting of polymer chains," Journal of Materials Chemistry, vol. 20, no. 10, pp. 19821992, 2010. 

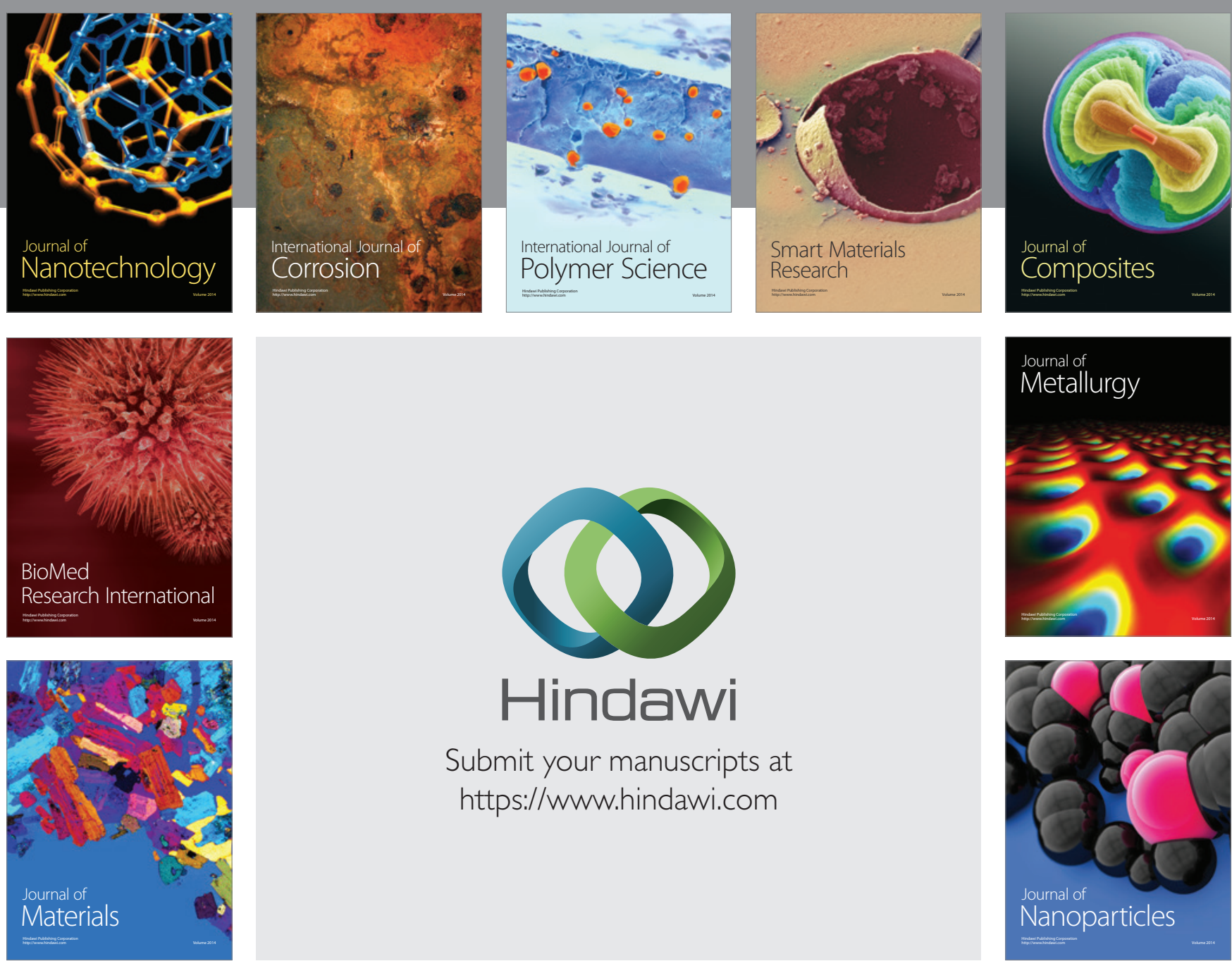

\section{Hindawi}

Submit your manuscripts at

https://www.hindawi.com

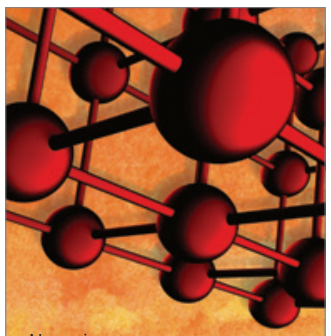

Materials Science and Engineering
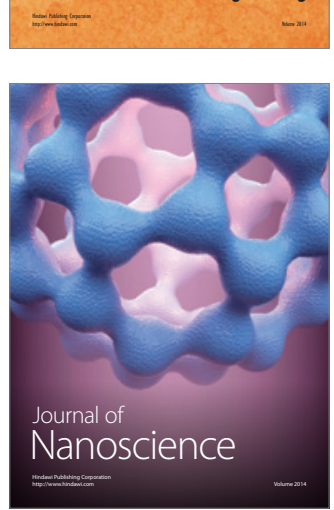
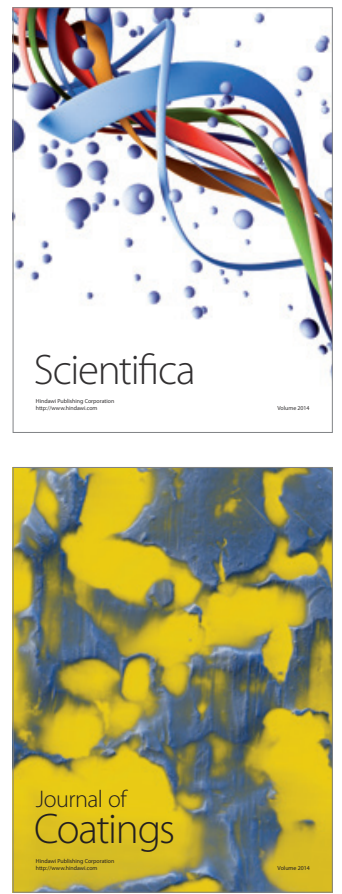
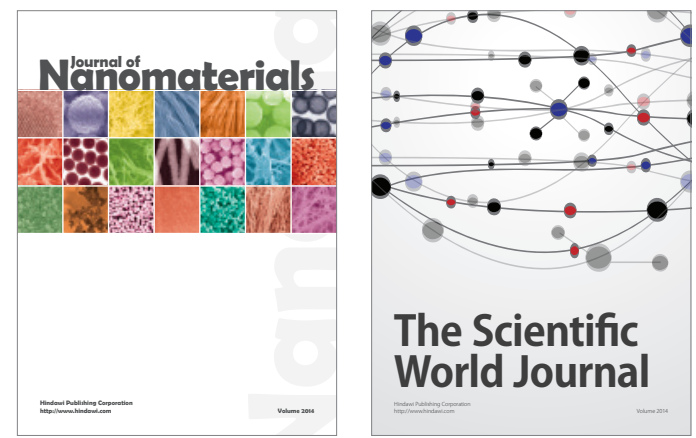

The Scientific World Journal
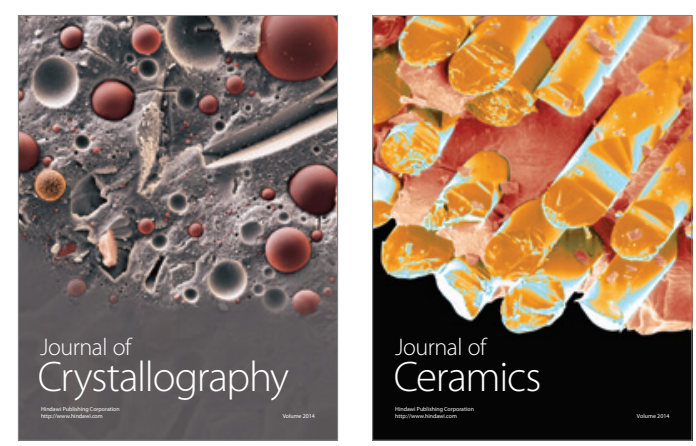
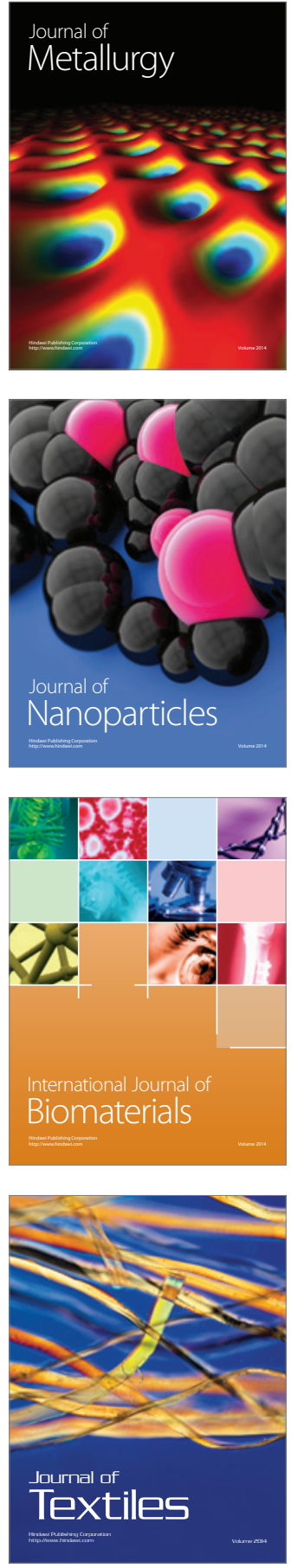